\section{№. 4239 January 27,1951 \\ Role of Dissolved Oxygen during the Inhibition of the Corrosion of Iron by Sodium Phosphate Solutions}

In was recently suggested by Mayne, Menter and Pryor $^{1}$ that dissolved oxygen is primarily responsible for the ability of $0 \cdot 1 \mathrm{~N}$ sodium hydroxide solution to inhibit the corrosion of iron, by virtue of a heterogeneous reaction with surface iron atoms to form a thin film of $\gamma-\mathrm{Fe}_{2} \mathrm{O}_{3}$. The present investigation was undertaken to determine the effect of dissolved oxygen on the corrosion-rate of iron totally immersed in solutions of sodium phosphate at different $p \mathrm{H}$ values.

The specimens, measuring $2.5 \mathrm{~cm} . \times 0.5 \mathrm{~cm}$, were cut from sheet $0.02 \mathrm{~cm}$. thick having the analysis : C, 0.005 ; $\mathrm{Si}, 0.0075$; S, 0.013 ; $\mathrm{P}, 0.003$; Mn, $0.007 ; \mathrm{Ni}, 0.018 ; \mathrm{Cr}, 0.02 ; \mathrm{Cu}, 0.003 ; 0,0.12$; $\mathrm{N}, 0.018$; H, 0.00009 per cent. Each specimen was degreased in benzene, abraded with $3 / 0$ emery paper, swabbed with acetone and weighed immediately before the beginning of the experiment. The solutions were prepared by mixing $M / 10$ solutions of monoand di-sodium phosphate to give a $p H$ range of $4 \cdot 5-$ $9 \cdot 1$ with a constant phosphate content.

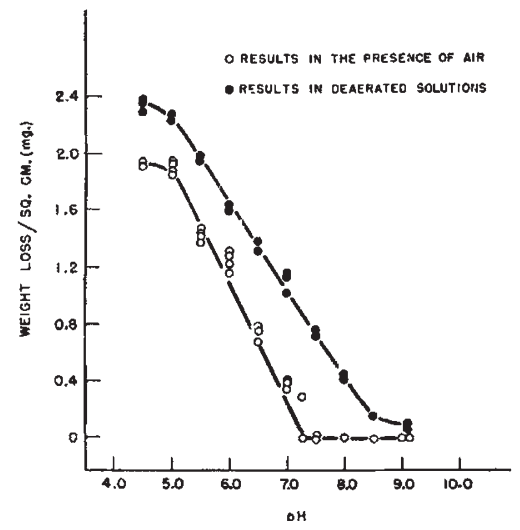

Fig. 1. Curves relating loss of weight per sq. cm., in flve days, $(a)$ in the presence of dissolved air, $(b)$ in deaerated solutions,
to the $p H$ of solutions of constant phosphate content at $25 \pm$ $0.05^{\circ} \mathrm{C}$.

In the first series of experiments, specimens were fully exposed to $5 \mathrm{ml}$. of solution saturated with air, and in free contact with the atmosphere, for five days, all runs being carried out in a water thermostat at $25 \pm 0.05^{\circ} \mathrm{C}$. At the end of the run the corrosion product was removed by 20 sec. exposure to $1: 1$ hydrochloric acid inhibited with 3 per cent 'Rodine'; exposure of a clean specimen to this solution for $300 \mathrm{sec}$. resulted in a loss of weight of less than $0.05 \mathrm{mgm}$. The specimens were then washed with water, dried with acetone, swabbed with benzene and finally re-weighed. The results (Fig. 1) show that passivity is obtained at $p \mathrm{H}$ values above $7 \cdot 25$.

Experiments under anaerobic conditions were carried out in the apparatus shown in Fig. 2, which involved the large change in gaseous solubility on freezing the solution. With the exception of the removal of dissolved air, other conditions were the same as in previous experiments in the presence of air. The specimen was contained in the limb $A$ and the solution in $B$. Deaeration consisted of five cycles in which the solution was first frozen with liquid nitrogen, the tap $D$ then opened to the vacuum system, at a pressure of $10^{-5} \mathrm{~mm}$. of mercury, for

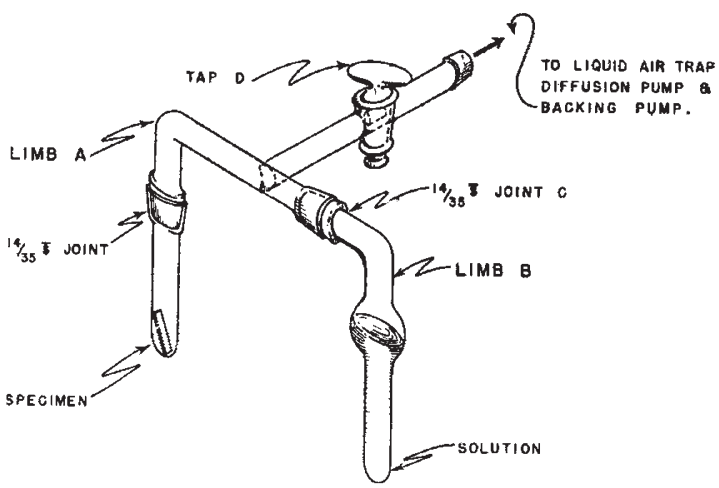

Fig. 2. Apparatus for experiments under anaerobic conditions

ten minutes and finally $D$ closed and the frozen solution in $B$ allowed to melt. During the fifth eycle, air was desorbed from the walls of the apparatus by gentle heating with a smoky flame. The solution was transferred into limb $A$ by rotating $\operatorname{limb} B$ about the $14 / 35$ standard-taper joint $C$; the apparatus was then removed from the vacuum line and placed in a thermostat at $25 \pm 0.05^{\circ} \mathrm{C}$. The results, also plotted in Fig. 1, show that once dissolved oxygen is removed from the phosphate it does not inhibit the corrosion of iron. The corrosion product in the absence of oxygen is a colourless tabular crystalline deposit.

Further work is being carried out on this subject and will be published at a later date.

M. J. Pryor

National Research Council,

M. COHEN Ottawa.

Aug. 3. ${ }^{1}$ Mayne, J. E. O., Menter, J. W., and Pryor, M. J., J. Chem. Soc. (in

\section{Electrical Conductivity of Borax Glasses containing Dispersed Gold}

IT will appear from previous communications ${ }^{1}$ regarding X-ray diffraction patterns of alkali metal salts, gold and platinum dispersed in borax glass that gold (with which we are concerned here) and also platinum exist as finely divided crystallites in the medium. The magnetic susceptibility of such samples has also been studied ${ }^{2}$.

The object of this note is to communicate certain interesting indications obtained in measuring the electrical conductivity of borax glass containing dispersed gold. The samples were prepared in the way similar to those described in the previous communications, and their electrical conductivities were calculated by obtaining the dimensions and the resistance of the sample from the drop of voltage across it and the current passing through it. The D.C. voltage (converted from A.c. mains through a transformer and a diode rectifier) used ranged from 150 to 450 volts. A standard resistance of 1 megohm had to be used in series with a 15-volt standard voltmeter to get the required range of voltage. The current through the sample was of the order of $10^{-6} \mathrm{amp}$. and was measured by a shunted d'Arsonval galvanometer having a figure of merit of the order of $10^{-10} \mathrm{amp}$. per mm. The percentages of gold in the samples were measured colorimetrically ${ }^{3}$. The results obtained are correct within \pm 5 per cent. 